Timo Meynhardt

\title{
Maß und Mitte reloaded: Eine visionslose Vision
}

Der Kapitalismus wird überleben, wenn er es schafft, Gemeinschaft und Gesellschaft immer wieder in eine für Mehrheiten erträgliche Balance zu bringen. Ansonsten nicht. Wenn die einen nicht mehr können und die anderen nicht mehr wollen, kommt - in Anlehnung an Lenin - etwas Neues.

Menschen leben zuallererst in ihren Familien, Beziehungen und sozialen Strukturen - kurz in ihren »Verbundenheiten «, die durch eine Wirtschaftsordnung geprägt, aber niemals vollständig determiniert werden können. Es ist die Qualität der »Verbundenheiten《 zwischen den Menschen, auf der jede Wirtschaftsordnung aufbaut und aus der sie ihre legitimierende Kraft bezieht. Von dort kommt der notwendige Rückhalt, der eine gesellschaftliche Ordnung erst überlebensfähig macht.

Natürlich setzen die Sozialverhältnisse den Rahmen für individuelle Entwicklung, aber diese sind selbst das Resultat psychischer Realitäten. Erstmals formuliert von Ferdinand Tönnies, einem der Gründungsväter der modernen Soziologie, kann dieser Gedanke nicht hoch genug geschätzt werden: »Alle sozialen Gebilde sind Artefakte von psychischer Substanz, ihr soziologischer Begriff muss zugleich psychologischer Begriff sein. ${ }^{\mathrm{I}}$ 
Für die heutige Soziologie »kommt dies der Aufdeckung einer vergessenen Problemsphäre gleich - der Fundierung der Sozialverhältnisse in Bewusstseinsdispositionen, welche als Verkörperung der `Gefühle, Triebe, Begierden $<$ dem vernunftorientierten Handeln nicht nur genetisch vorausgehen, sondern dieses selbst erst aus sich entlassen. $\ll^{2}$

Mit dieser sozialtheoretisch inspirierten Perspektive ist auf den Punkt gebracht: Die Zukunft des Kapitalismus in all seinen Spielarten (mindestens: amerikanisch, chinesisch, europäisch) wird davon abhängen, inwieweit die Umsetzung seiner Grundprinzipien, insbesondere der freie Wettbewerb, das Privateigentum und die Steigerungslogik der Kapitalakkumulation positiv auf die Erfüllung menschlicher Grundbedürfnisse einwirkt und damit nachweislich einen Gemeinwohlbeitrag erbringt. Jede ambitionierte Kapitalismustheorie benötigt eine Vorstellung zum Wesen des Menschen, seinen >Gefühlen, Trieben, Begierden $<$ und davon, wie auf dieser Basis ein Gemeinweisen funktionieren kann.

Es ist, ob man es will oder nicht, der psychologischsubjektive Faktor, der darüber entscheidet, ob und in welchem Ausmaß jede Art von Systemtransformation stattfindet oder nicht. Oder anders formuliert: Die Zukunft des Kapitalismus entscheidet sich gerade nicht primär in der ökonomischen Sphäre, sondern in seiner Fähigkeit, soziale Bindungen innerhalb und zwischen Gruppen zu stärken bzw. immer wieder in eine erträgliche Balance zu bringen.

Das wissen auch andere: Im Januar 2018 hat Larry Fink, der Gründer, Aufsichtsrats- und Vorstandsvor256 
sitzende des weltgrößten Vermögensverwalters BlackRock, die Unternehmen aufgefordert, einen »Sense of Purpose « zu entwickeln und ihr Geschäft stärker am gesellschaftlichen Nutzen auszurichten. So vielfältig die Motive für diesen Aufruf sein mögen, in jedem Fall verweist er auf die gesellschaftlich zunehmend lauter gestellte Frage nach der Akzeptanz der kapitalistischen Wirtschaftsordnung bzw. auf die wiedererwachte Einsicht des gesellschaftlichen Eingebundenseins jedes unternehmerischen Handelns.

Zum Beispiel erhalten die immer wieder diskursdominierenden Fragen nach mehr/weniger Markt, mehr/ weniger Digitalisierung oder mehr/weniger Globalisierung ihre eigentliche systemische Relevanz durch ihren Einfluss auf die Qualität gemeinschaftlicher bzw. gesellschaftlicher Verbundenheiten. Anders formuliert: Die Zukunft des Kapitalismus wird davon abhängen, ob es gelingt, jene anthropologischen Grundkonstanten des Menschen zur Geltung kommen zu lassen, die auf das Miteinander, Solidarität und Mitgefühl ausgerichtet sind.

\section{Die Jahrhundertidee von Gemeinschaft und Gesellschaft}

Sozialverhältnisse ermöglichen unterschiedliche Ausprägungsformen von Individualität und Sozialität. Wer etwa das Wechselspiel von »Ich « und »Wir«, das »Wir im Ich « verstehen möchte, findet in den Sozialwissenschaften unterschiedlichste Anknüpfungspunkte. Am Anfang soziologisch-psychologischer Theoriebildung 
stehen Ferdinand Tönnies' Überlegungen, welche im 20. Jahrhundert so gut wie alle bedeutsamen Soziologen in diesem Feld geprägt haben.

Er hat dabei ein Gedankengebäude errichtet, welches zwar Fragment geblieben ist, für die Frage zur Überlebensfähigkeit des Kapitalismus jedoch durchaus reizvoll und wegweisend ist. Dies vor allem deshalb, weil Tönnies sich einer einseitigen Parteinahme für eine rationalistische Sicht des vernunftbegabten Menschen (»Gesellschaft«) genauso verweigert wie dem anderen Extrem einer aufklärungsfeindlichen, fortschrittsskeptischen Haltung (»Gemeinschaft«).3 Mit seiner Unterscheidung zwischen »Gemeinschaft « und »Gesellschaft $\ll 4$ hat Tönnies in erster Line fundamentale Mechanismen menschlichen Zusammenlebens in einem Gemeinwesen beschrieben, die uns heute helfen können, Phänomene wie »soziale Spaltung«, »Filterblasen« oder »Entfremdung «, aber auch das Aufkommen neuer sozialer Bindungsformen zu verstehen. An dieser Stelle sei darauf hingewiesen, dass jeweils auch der Plural gemeint ist: Gemeinschaften und Gesellschaften. Die Substantivierungen dienen Tönnies primär zur prägnanten Benennung unterschiedlicher Bindungsformen zwischen Menschen.

Eines ist für Tönnies dabei sehr klar: Bindungsrelevant sind nicht allein rationale Gesichtspunkte oder logisch bessere Argumente, sondern vor allem auch rational nicht fassbare Emotionen und Motivationen. Diese lassen sich nicht mit Argumenten »überzeugen «. In der damaligen Sprache von Tönnies ist es immer »... ein bestimmtes Verhältniß, in welches der empfundene innere Gesammtzustand zu diesen besonderen

258 
Empfindungen sich setzt. Denn jener ist das absolute A priori, und er kann nur gedacht werden als die Existenz der gesammten Natur durch allgemeine und dunkle Beziehungen auf sich involvirend, von welchen dann einige durch Entwicklung und Actionen des Gehirnes und der Sinnesorgane, d.h. des verstehenden (davorstehenden) Geistes, allmählich klarer und deutlicher werden. $\ll$

Für Tönnies sind soziale Gebilde (wie auch unsere Wirtschaft eines ist) ganz wesentlich durch unsere biologisch-psychologische Entwicklung mitbestimmt. ${ }^{6}$ Aus der Einheit der psychischen Realität von >Gefühlen, Trieben und Begierden< und dem darin eingeschlossenen Denken bilden sich zunehmend differenziertere Erfahrungswelten (Tönnies spricht von »Willensformen«) heraus. Diese sind die eigentliche Grundlage für die Formen sozialer Beziehungen (»Verbundenheiten«). Es wirken dann zwei antreibende, komplementäre Mechanismen, die eben nicht einfach ein Ganzes bilden, sondern in einem spannungsgeladenen Verhältnis zueinander stehen. Der erste wird mit dem Begriff der Gemeinschaft umschrieben und zielt auf das »reale und organische Leben« und damit auf sinnliche Erkenntnis, leibliche Erfahrung und emotionale Verbundenheit.

Aus dieser wächst die ideelle mechanische Bildung heraus - mit dem Begriff der Gesellschaft beschrieben. Bei Tönnies heißt es: »In Gemeinschaft mit den Seinen befindet man sich, von Geburt an, mit allem Wohl und Wehe daran gebunden. Man geht in die Gesellschaft wie in die Fremde. «7 Es ist natürlich ein großer Fortschritt, sich aus den Bindungen des unmittelbaren Umfelds zu lösen. Die »Fremde« muss auch nichts Bedrohliches 
darstellen (zum Beispiel »Stadtluft macht frei«). Vielmehr speist sich daraus die Kraft für Neues.

Die ordnende Kraft in der Gemeinschaft erwächst aus den »Verbundenheiten «, die in unmittelbaren menschlichen Beziehungen (Verwandtschaft, Nachbarschaft, Freundschaft) wirken. Entscheidend ist hier die erfahrungsmäßige Einheit von Verstand und Gefühl. In der Gemeinschaft sind Menschen durch gute/schlechte Sitten, Alltagsvernunft oder auch durch den "gesunden Menschenverstand « miteinander wesentlich verbunden. Gemeinsam ist diesen Bindungsformen, dass sie auch ganz ohne von außen herangetragene Vorschriften oder Regelungen funktionieren.

Der menschliche Geist besitzt jedoch die Fähigkeit, aus der unmittelbaren sinnlichen Erfahrung herauszutreten. Mit zunehmender Ausdifferenzierung und Reflexivität entstehen so neue Formen der »Verbundenheit «. Diese lösen sich aus der ursprünglichen instinkthaft-natürlichen, später auch aus der verstandesmäßigen Einheit von Denken und Fühlen. In der bio-psycho-sozialen Entwicklung schieben sich immer neue komplexere Ebenen der Reflexion und geistige Operationen zwischen die Erscheinungsweisen sinnlich geprägter Natürlichkeit. Die gedankliche Bildung von »Begriffen", die Entwicklung von »Absichten «, das Setzen von "Zwecken « sind Ergebnis dieser neuen psychischen Realitäten und erschließen einen weiteren, neuen Mechanismus der »Verbundenheit«, nämlich den der verstandesmäßig bewusst geschlossenen Kontrakte.

Es entstehen so immer neue mentale Strukturen, mit deren Hilfe sich neue Erkenntnisformen herausbilden. 
Das Element des Abstrakten entfaltet dabei eine eigenständige Wirkmacht und eröffnet neue Handlungsmöglichkeiten. Wir können Konsequenzen unseres Handelns vorwegnehmen und durchspielen; wir können bisher unverbundene Tatsachen ins Verhältnis setzen oder neu verbinden. Diese geistigen Leistungen sind gleichzeitig Voraussetzung für die Etablierung neuer Sozialformen und werden umgekehrt zugleich von diesen getragen.

Das Wesen von Gesellschaft (im Gegensatz zur Gemeinschaft) liegt damit in der von Subjektivität und Emotionalität entkoppelten abstrakten Regelwelt. Eine damit ermöglichte Formalisierung der Tauschbeziehungen im Wirtschaftsleben ist die Grundlage von Handelsbeziehungen. Insofern ist gerade auch die kapitalistische, d.h. auf den Kapitalfluss angewiesene, Wirtschaftswelt auf Vergesellschaftungsprozesse (z. B. in Form eines funktionierenden Rechtsstaates) im Sinne von Tönnies angewiesen. Erst die Herausbildung von formalisierten gesellschaftlichen Sozialformen (Privatrecht: Tausch, Vertrag, Handel; Öffentliches Recht: Bewilligungen, Verbote, Abgaben etc.) und ihren wirtschaftlichen Institutionen (bis hin zu anonymen Kapitalgesellschaften und staatlichen Interventionen in den Wirtschaftsprozess) ermöglicht verlässliche Rahmenbedingungen für die Wirtschaft über die Grenzen der Gemeinschaft hinaus. Aber auch jede Zweckrationalität ökonomischen Handelns und das Kalkül des Tauschwertes müssen auf die sie tragenden menschlichen Motivationen bauen können. Sonst bröckeln Glaubwürdigkeit und Legitimation. Wirtschaftssysteme und ihre Abläufe sind eben nicht aus sich selbst heraus und 
objektiv erklärbar, sondern immer Resultat »menschlichen Willens « und insofern gewollt.

Folgt man dem Gedanken, dass Sozialverhältnisse durch Bewusstseinsdispositionen fundiert sind, durch diese angetrieben und überhaupt erst ermöglicht werden, dann kommt man einem etwaigen Krisenmechanismus auf die Spur: Je weiter die beiden Sphären von Gemeinschaft und Gesellschaft aufgrund zunehmender ökonomischer, sozialer und technischer Komplexität auseinanderdriften, desto schwieriger wird es, zu »verstehen«, um was es eigentlich geht. Mehr Komplexität kann nicht allein durch besseres »Erklären « bewältigt werden, sondern sie erzwingt zwangsläufig eine Sinnstiftung über emotional-motivational verankerte Werte. Diesen kommt dann eine Ordnungsfunktion zu, weil sie eine unabdingbare Vereinfachung von Komplexität und damit Orientierung ermöglichen.

\section{Fahren im Nebel}

In mancher Hinsicht müssen Entscheidungsträger in der Wirtschaft oftmals wie Autofahrer im dichtesten Nebel agieren - nicht wissend, ob sie auf der richtigen Spur sind, riskierend, dass es zum Frontalzusammenstoß mit dem Gegenverkehr kommt - und trotz allem weiterfahrend. Im Gegensatz zum tatsächlichen Straßenverkehr ist es hier gerade kein verlässlicher Indikator, auf der falschen Spur zu sein, wenn einem mehrere hundert Fahrzeuge entgegenkommen. Dies ist an sich kein neues Phänomen; neuartig sind jedoch Hebelwirkung und Geschwindigkeit in technisch hoch 
entwickelten und integrierten Handelssystemen und Wirtschaftskreisläufen.

Wir sehen uns heute mit einer Ansammlung komplexer Situationen konfrontiert, und im Feld der Komplexität gilt, dass so mancher Lenkungseingriff dem Tanz der Regenmacher gleicht. Wir hören und sagen es nicht gerne, und doch lässt es sich nicht bestreiten: Egal, ob Ökonomen, Soziologen oder Psychologen, wir sind gnadenlos überfordert. Die gute Nachricht ist, dass die Überforderung chronisch ist. In der Krise sehen wir dies besonders deutlich. Und deshalb tun wir gut daran, in unseren Überlegungen und Entscheidungen ebenso bescheiden wie differenziert vorzugehen.

Dass wir im Dunkeln tappen, ist mithin nicht nur unserem Unvermögen zuzuschreiben, sondern hat seine Ursache in der Weise der (Dys-)Funktion unserer modernen Gesellschaften. Es handelt sich dabei um den Trend der Überführung von Sachverhalten, Geschäftsbeziehungen, Risikoabwägungen oder Produktideen in anspruchsvolle geistig-abstrakte Zusammenhänge, die wir nicht mehr durchschauen, in rechtliche Normierungen, unnötig komplizierte Modelle und Theoriengebäude, deren Rückübersetzung in die Welt des gesunden Menschenverstandes oft kaum mehr möglich ist. Die großartige Fähigkeit des menschlichen Geistes, durch Abstraktion aus der unmittelbaren Erfahrungswelt herauszutreten, schlägt schnell in Unübersichtlichkeit um, weil die emotional-motivationale (Re-)Integration immer schwerer gelingt.

Ich hatte eingangs vollmundig von einem »Jahrhundertgedanken « gesprochen. Die nur in der oberflächlichen Betrachtung schematisch wirkende Trennung 
von Gemeinschaft und Gesellschaft ist deshalb fundamental, weil sie eine zentrale ambivalente Erfahrung zwischenmenschlichen Zusammenlebens in der Moderne in origineller Weise aufgreift. Auf der einen Seite stehen unmittelbar persönliche Beziehungen, in denen der Einzelne sich emotional-motivational eingebunden und verwurzelt fühlt. Auf der anderen Seite finden sich als äußerlich, nicht überschaubar und fremd erlebte gesellschaftlich-anonyme Abläufe - Verhältnisse, die nicht beeinflussbar scheinen. Trotz ihrer Unterschiedlichkeit sind beide »Verbundenheiten « Ergebnis von realen Entwicklungen mit den ihnen innewohnenden psychologischen Prozessen und beide müssen sich stets aufs Neue gegenüber einer ganzheitlichen Bewertung (»empfundener innerer Gesammtzustand «) bewähren.

Es war schon für Tönnies völlig klar, dass es nicht die Gesellschaft/Gemeinschaft gibt, sondern viele Gemeinschaften und Gesellschaften. Im Gegensatz zu den unterschiedlichsten Etiketten der Gegenwartsbeschreibung, z. B. Risiko-, Freizeit-, Multioptions- oder Wissensgesellschaft, geht es bei Tönnies um Antworten auf die Frage, wie unterschiedliche Arten von »Verbundenheiten « in einem Sozialverband entstehen und sich wie chemische Elemente immer neu zusammensetzen. Dabei ist es unerheblich, ob man diese Fragen etwa in einzelnen »Parallelgesellschaften « oder »virtuellen Gemeinschaften « untersucht. Im Kern steht immer die Frage im Mittelpunkt, wie Verbindlichkeit in zwischenmenschlichen Beziehungen entsteht.

In der Ausgestaltung der Idee thematisiert Tönnies dabei - teilweise in Vorwegnahme späterer Erkenntnisse der Psychologie - die oftmals getrennten Sphären 
von ganzheitlicher Erfahrung und (re)integrationsbedürftigem Wissen. Deren erlebnismäßiges Auseinandertreten und der daraus stets aufs Neue erwachsende Integrationsbedarf werden bei Tönnies nicht nur als individuelles, sondern als soziales Problem formuliert.

Das Auseinandertreten von Gemeinschaft und Gesellschaft bewirkt dann Entfremdungserfahrungen (»sich nicht mehr verstanden fühlen «, »soziale Fragmentierung «, »die Welt antwortet nicht mehr« usw.). Für den Soziologen Hartmut Rosa besteht eine Lösung darin, neue Resonanzerfahrungen zu suchen. ${ }^{8}$ Der Soziologe Armin Nassehi dagegen wirbt für einen besseren »Umgang mit und die Nutzung von Perspektivendifferenz «.9 Gerade Letzteres dürfte sich allerdings vor dem Hintergrund einer neuen Balanceanforderung zugunsten von Gemeinschaftserfahrungen in überschaubaren Gruppen als naiv erweisen. Wenn das Pendel $\mathrm{zu}$ weit in Richtung von komplexitätssteigernder Vergesellschaftung ausgeschlagen hat, kann eine weitere Steigerung von Perspektivenakzeptanz kaum - so wünschbar dies sein mag - ein Erfolgsrezept sein. Das aktuelle Erstarken populistischer Strömungen, bei denen die Gemeinschaftsorientierung (aktuell besonders über Konzepte wie nationale Identität oder Heimat) betont wird, spricht da eine klare Sprache. Die Herausforderung besteht darin, legitimationsfähige Angebote $\mathrm{zu}$ machen, wie die Balance zwischen Gemeinschaft und Gesellschaft in einer produktiven und letztlich für Mehrheiten erträglichen Spannung gehalten werden kann.

Vielleicht kommen aber auch tragfähige Antworten aus der im Kapitalismus angelegten Innovationsorien- 
tierung. Der unternehmerische Ansatz, stärker gemeinschaftsorientierte Produkte und Dienstleistungen anzubieten, führt schon heute $\mathrm{zu}$ erfolgreichen Geschäftsmodellen - seien es die Aufwertung von Regionalität im Lebensmittelhandel, Community-orientierte Marketingansätze und nicht zuletzt Ideen der Sharing Economy. Es liegt durchaus auch im Geschäftsinteresse der Unternehmen, durch ihre Produkte und Dienstleistungen an tragfähigen »Verbundenheiten« mitzuwirken. Dies betrifft aber auch die interne Unternehmensorganisation selbst. Auch dort ist sinnvolle Vereinfachung das Gebot der Stunde, um neue Formen der Verbundenheit auf Gemeinschaftsebene zu ermöglichen. Insofern sind neue Arbeitsformen (agil, holokratisch usw.) als Versuche zu interpretieren, die kapitalistische Produktionslogik in immer neue Vergemeinschaftungsformen einzubinden bzw. soziale Bedürfnisse damit zu verbinden. Erinnert sei hier auch noch einmal an die eingangs zitierte Forderung nach einem social purpose von Larry Fink, die eben auch als Aufruf zur Entwicklung innovativer Ansätze zur Stärkung von »Verbundenheiten « gelesen werden kann. Längst zeigt die Praxis, wie Unternehmer versuchen, die Steigerungslogik der Kapitalakkumulation in den Dienst neuer gemeinwohldienlicher Wertschöpfung zu stellen.

Allerdings: Inwieweit eine dadurch an verschiedensten Stellen in Gang gesetzte Selbsttransformation des Kapitalismus zur Überwindung von Widersprüchen, Lösung von Konflikten und nicht zuletzt sozialer Stabilität beiträgt, bleibt abzuwarten. Es ist auch Vorsicht geboten, in einem komplexen, nicht überschaubaren 
Wirtschaftssystem Einzelbeispiele zu verallgemeinern bzw. einer Steuerungsphantasie Vorschub zu leisten. Selbst Wolfgang Streeck, ein prominenter und sehr fundierter Kapitalismuskritiker, gesteht ein, dass der gegenwärtige Zustand des Kapitalismus ein emergenter ist, d. h. »von den beteiligten Akteuren weder geplant noch gewollt werden muss $\ll .{ }^{10}$ In einem selbstorganisierenden System (und anders kann man heute kaum noch sinnvoll über die kapitalistische Ordnung sprechen), haben wir keinen Überblick mehr.

\section{Lob der kleinen Schritte in Richtung Gemeinwohl}

Nicht allein vom Wohlwollen der lokalen Gemeinschaft abhängig zu sein, sondern sich auf rechtliche Verbindlichkeiten berufen zu können und mit Hilfe des abstrakten Mediums »Geld « auch andernorts sich etwas aufzubauen, ist Teil dessen, was Tönnies mit Vergesellschaftung meint. Das Element des Abstrakten, das von der unmittelbaren emotionalen Beziehung Losgelöste, war ursprünglich ein großer Freiheitsgewinn und Fortschrittsmotor. Die aktuellen Krisenerscheinungen zeigen uns jedoch, dass die alte Balance zwischen dem Konkreten (Gemeinschaft) und dem Abstrakten (Gesellschaft) verlorengegangen ist.

Dieses Auseinanderklaffen scheint auch der entscheidende Nährboden für künftige Krisen und wiederkehrende Störungen unseres Wirtschaftssystems zu sein. Es ist nur eine Frage der Zeit, bis in der vernetzten Dynamik einer globalen Wirtschaft der Motor aufgrund der hochabstrakten Finanzströme wieder ins Stottern gerät. 
Wer sich dieser Perspektive befleißigt, kommt zu dem Schluss: Wir leiden nicht unter zu viel freier Marktwirtschaft oder unter zu viel Sozialstaat. Wir leiden vielmehr unter der Logik einer exzessiven Vergesellschaftung, die dem gesamten Wirtschaften, mithin auch dem modernen Finanzkapitalismus, eigen ist und durch ihn weiter forciert wird. Es ist also weniger das Profitstreben als die systemisch eingebaute Abstrahierung, Objektivierung und Verdinglichung menschlichen $\mathrm{Zu}$ sammenlebens, der wir bisher keine wirkungsvollen Konzepte entgegenzustellen haben. Denn klar ist: Ein Zurück in »alte Zeiten« gibt es nicht.

Drei Schlüsse bieten sich hier an. Erstens müssen wir die Kraft aufbringen, durch klare, aber einfache Regeln im politischen Bereich den Grad der »Vergesellschaftung « im hier besprochenen Sinne nicht weiter voranzutreiben. Oder besser: wir müssen Anreize zur Vereinfachung setzen. Wir dürfen die Dinge nicht trivialisieren, aber eine weitere Komplexitätssteigerung wird die Probleme nicht lösen. Folgen wir dem vorgetragenen psychologisch-soziologischen Argument, so können auch technische Innovationen (aktuell die Digitalisierung) uns kaum weiterhelfen, solange wir im abstrakten Raum agieren, welchen wir nicht emotional besetzen können. Zweitens müssen wir unser Bedürfnis zügeln, große Visionen zu entwerfen. Der Verlauf der Geschichte zeigt, dass wenige Dinge wirklich vorhersagbar sind - auch nicht in einem halbwegs übersichtlichen System. Wir tun deshalb gut daran, dem »Piecemeal-Approach « im Sinne des Philosophen Karl Popper wieder mehr Wertschätzung entgegenzubringen - kleine Schritte, im tastenden Verfahren, stets bereit 
zur Umkehr. Wir müssen auch unsere Erwartungen an die Politik zurückschrauben - niemand kann so einfach einen oder zwei Hebel umlegen. Unser Wirtschaftssystem ist in all seinen gesellschaftlichen Verflechtungen viel zu vernetzt, als dass kraftvolle monokausale Interventionen nicht sogleich die Gesamtstabilität bedrohen könnten - die unbeabsichtigten Nebenwirkungen fallen mittlerweile stärker ins Gewicht als die beabsichtigten. Woran sollen sich Entscheidungsträger denn nun orientieren, wenn alles so schrecklich kompliziert und komplex ist?

Als dritte und wesentlichste Konsequenz folgt: Orientiere dich am Gemeinwohl und prüfe dein Handeln am Maßstab der Gemeinwohlverträglichkeit! Allein schon aus Gründen der wechselseitigen Abhängigkeit in einem gemeinschaftlich-gesellschaftlichen Zusammenhang ist dieser Imperativ rational. Es ist nur ein schwaches Gegenargument, dass die Idee des Gemeinwohls antiquiert sei, weil darunter alle etwas anderes verstünden. Sicher bedarf es der ständigen Vorsicht, dass mit einer solchen Rhetorik nicht - wie historisch geschehen - autoritärer Missbrauch getrieben wird. Doch ist der aktive Beitrag zur Stärkung des Gemeinwesens bzw. die Verhinderung von dessen Schädigung die entscheidende Legitimation für unsere freiheitliche Ordnung. Nichts ist kurzsichtiger als ein Freiheitsgedanke, der nicht erkennt, dass es keine voraussetzungslose menschliche Existenz gibt. Gemeinwohl ist als eine Bedingung der Möglichkeit gelingenden Lebens zu betrachten - oder etwas plakativer: »Ohne Gemeinwohl keine Freiheit«. Gemeinwohl wird zur Grundbedingung menschlicher Selbstentfaltung. 
Auch wenn es schwer zu ertragen ist: Unser Verstand steht uns im Wege, und wir tun gut daran, sein Scheitern in Rechnung zu stellen. Wenn wir also eine freie Gesellschafts- und Wirtschaftsordnung erhalten wollen, bedarf die Tendenz zur Abstrahierung der Geschäftsabläufe und -beziehungen aus den unmittelbaren Lebensverhältnissen einer kulturell akzeptierten, emotional-motivationalen Rückbindung. Das Gemeinwohl scheint mir da die sinnvollste, historisch gewachsene regulative Idee zu sein. Um es maximal simpel zu sagen: Der »gesunde Menschenverstand « muss als Richtschnur für menschliches Entscheiden und Handeln neu entdeckt werden. Kritik ist immer da angebracht, wo der »gesunde Menschenverstand « nicht in der Lage ist, das Argument oder die Lösung nachzuvollziehen.

Die Beschäftigung mit entscheidungsunterstützenden guten Gefühlen (ich sage bewusst nicht: mit guten Gründen) sollte nicht nur im Berufsleben, sondern auch in den primären und sekundären Bildungsinstitutionen zu einem wichtigen Gut werden. Denn damit lässt sich die Bereitschaft stärken, sich mit dem damit verbundenen liberalen Menschenbild zu beschäftigen, und lassen sich Anreize setzen, die dies honorieren. Was man nicht kann, ist, Werte zu diktieren oder mit Zwang durchzusetzen.

Halten wir also fest: Das Funktionieren einer kapitalistisch geprägten Wirtschaftsordnung, in der » $\mathrm{Ka}-$ pital « und »Risiko e eine entscheidende Rolle spielen, ist zwingend auf eine Orientierung am Gemeinwohl angewiesen. Ein solches Prüfkriterium zwingt zu einem Abgleich zwischen allen produktiven und destruktiven Kräften der Abstraktion, welche die Errungenschaften

270 
der einzelnen Varianten des Kapitalismus erst möglich machen. Gefragt sind eine Gangart der kleinen Schritte und der Mut zur Vereinfachung. Ohne eine solche Besinnung auf »Maß und Mitte "(Wilhelm Röpke) ${ }^{\mathrm{II}}$ steht die Lebensfähigkeit der gesellschaftlichen Ordnung auf dem Spiel. Die Gemeinwohlorientierung stellt dabei einen Kompass bereit, die Gemeinschaftserfahrung nie einseitig zu Lasten der Gesellschaftserfahrung aufzugeben und umgekehrt, immer wieder nach der produktiven Kopplung zwischen beiden zu suchen. Es ist hierbei von zentraler Bedeutung, nicht auf eine Harmonie zu setzen, sondern den Dualismus anzuerkennen und das unauflösbare Spannungsverhältnis kreativ zu nutzen.

\section{Auf dem Weg zu einer visionslosen Vision}

Die formulierte »Überlebensbedingung « für den Kapitalismus ist zutiefst am Gedanken der Gemeinwohlverträglichkeit orientiert. Hier sind immer wieder Innovationen gefragt, um die Balance zwischen Gemeinschaft und Gesellschaft stets neu auszutarieren. Mit den Ideen von Ferdinand Tönnies verfügen wir dabei über einen Erklärungsansatz, der die Abhängigkeit der kapitalistischen Produktionsweise von einer Gemeinwohlorientierung auf den Punkt bringt. Vor allem aber zeigt er auf, wo Unternehmen heute ansetzen können, um ihre Akzeptanz in der Bevölkerung zu erhalten bzw. zu steigern.

Angesichts des selbstorganisierenden Charakters komplexer Systeme ist allerdings jede Art von Vision von Anbeginn zum Scheitern verurteilt, wenn sie sich 
den großen Wurf vornimmt. Ganz im Sinne des erwähnten »Piecemeal-Approaches « kommt es darauf an, neue Formen der Verbundenheiten im Experiment zu wagen und tragfähige Lösungen zu entdecken. Das damit verbundene "Durchwursteln « ist eben gerade keine Schwäche, sondern ein Eingeständnis an die durch uns Menschen erzeugte Komplexität.

Dies bedeutet nicht eine Absage an individuelle Ziele und Zukunftsvorstellungen, die dem Einzelnen Kraft und Richtung geben und ihm als Lebensantrieb dienen. Visionslose Vision meint auch nicht keine Vision. Eher zielt der Begriff auf die Bereitschaft, sich schonungslos der Begrenztheit individueller Handlungsmacht zu stellen und sich gleichzeitig immer wieder am größeren Ganzen auszurichten. Diesen scheinbaren Widerspruch zu leben, dürfte damit beginnen, ihn zunächst überhaupt als echte Herausforderung $\mathrm{zu}$ erkennen und anzunehmen.

Der einhergehende Gestaltungs- und Wirkungsanspruch einzelner Akteure ist dann von Bescheidenheit und von Demut gekennzeichnet, wie es seit den Stoikern empfohlen wird: sich gelassen nur auf jene Dinge zu konzentrieren, die man wirklich ändern kann und diese mutig anzupacken. Das ist dann vielleicht auch der Kern einer visionslosen Vision, die tragfähige Verbundenheiten zugunsten des Gemeinwohls im Blick hat und sich nicht verführen lässt von uneinlösbaren Zukunftsversprechen. Indem sich der Kapitalismus in dieser Weise zu sich selbst bekennt, hat er eine Überlebenschance. 


\section{Anmerkungen}

I Ferdinand Tönnies, Gemeinschaft und Gesellschaft. Grundbegriffe der reinen Soziologie. 8. Auflage von I 935; 4. unveränderte Auflage, Darmstadt I979/2005, S. XXXIV.

2 Peter-Ulrich Merz-Benz, Tiefsinn und Scharfsinn. Ferdinand Tönnies' begriffliche Konstitution der Sozialwelt, Frankfurt am Main i 995 .

3 Cornelius Bickel und Ferdinand Tönnies, Soziologie als skeptische Aufklärung zwischen Historismus und Rationalismus, Opladen r99I.

4 Tönnies (Anm. I).

5 Tönnies (Anm. I), S. XVI.

6 Nachfolgende Textabschnitte gehen in Teilen zurück auf den Beitrag von Timo Meynhardt, Management zwischen Main Street und Wall Street. In: Management - eine gesellschaftliche Aufgabe, Baden-Baden 2010, S. 19-45 und den Beitrag von Timo Meynhardt, »Mass und Mitte«, Schweizer Monat I000, Oktober 20I 2, S. 73-78.

7 Tönnies (Anm. I), S. 3.

8 Hartmut Rosa, Resonanz: Eine Soziologie der Weltbeziehung, Frankfurt am Main 2018.

9 Armin Nassehi, Die letzte Stunde der Wahrheit. Kritik der komplexitätsvergessenen Vernunft (kursbuch edition), Hamburg 2017, S. 209.

Io Wolfgang Streeck, Gekaufte Zeit: Die vertagte Krise des demokratischen Kapitalismus, Berlin 2017, S. 20.

I I Wilhelm Röpke, Maß und Mitte, Erlenbach/Schweiz I950. 